\title{
Warm temperature stimulus suppresses the perception of skin wetness during initial contact with a wet surface
}

\author{
D. Filingeri ${ }^{1}$, B. Redortier ${ }^{2}$, S. Hodder ${ }^{1}$ and G. Havenith ${ }^{1}$ \\ ${ }^{1}$ Environmental Ergonomics Research Centre, Loughborough Design School, Loughborough University, Loughborough, UK and \\ ${ }^{2}$ Thermal Sciences Laboratory, Oxylane Research, Villeneuve d'Ascq, France
}

\begin{abstract}
Background/purpose: In the absence of humidity receptors in human skin, the perception of skin wetness is considered a somatosensory experience resulting from the integration of temperature (particularly cold) and mechanical inputs. However, limited data are available on the role of the temperature sense.

Methods: Wet and dry stimuli at $4^{\circ} \mathrm{C}$ and $8^{\circ} \mathrm{C}$ above local skin temperature were applied on the back of seven participants (age $21 \pm 2$ years) while skin temperature and conductance, thermal and wetness perceptions were recorded.

Results: Resting local skin temperature was always increased by the application of the stimuli $\left(+0.5-+1.4^{\circ} \mathrm{C}\right)$. No effect of stimulus wetness was found on wetness perceptions $(P>0.05)$. The threshold (point ' -2 slightly wet' on the wetness scale) to identify a clearly perceived wetness was never
\end{abstract}

reached during any stimulations and participants did not perceive that some of the stimuli were wet. Overall, warm temperature stimuli suppressed the perception of skin wetness.

Conclusion: We conclude that it is not the contact of the skin with moisture per se, but rather the integration of particular sensory inputs (amongst which coldness seems dominant) which drives the perception of skin wetness during the initial contact with a wet surface.

Key words: skin wetness - temperature - thermoreceptors perception

(C) 2014 John Wiley \& Sons A/S. Published by John Wiley \& Sons Ltd

Accepted for publication 5 January 2014
$\mathrm{T}$ HE PERCEPTION of skin wetness is a complex somatosensory experience which seems to result from the integration of temperature and mechanical (i.e. pressure) inputs (1-3). To date, a hygroreceptor has never been identified on the human skin (4). Therefore, it has been suggested that human beings learn to perceive the wetness experienced when their skin is in contact with a wet surface, when a liquid is touched, or when sweat is produced (3). The thermal and mechanical inputs which result from the physical processes occurring when the skin is in contact with moisture (i.e. heat transfer and mechanical interactions between the skin and the environment) could be integrated and combined at different anatomical levels through specific multisensory pathways (5). However, although the interaction between thermal and mechanical inputs seems to be the principal inducer of the perception of skin wetness (1-3), to date it is unclear which sensory modality is dominant in driving this perception.

The thermal sense might play a significant role in this perception. We have recently shown that exposing the skin to cold-dry stimuli (resulting in cooling rates similar to the ones occurring during the evaporation of water from the skin) can evoke an illusion of local skin wetness $(6,7)$. This indicated that in particular situations, individuals seem to associate local coldness with local skin wetness. These recent findings have opened an interesting question: if skin wetness might be primarily driven by coldness, would individuals be able to perceive local skin wetness if exposed to a local warmwet stimulus during which no coldness is experienced? It might be hypothesised that in that case, the ability to perceive local skin wetness would depend upon the mechanical cues available. Every day experience indicates that we are able to perceive the wetness of a warm liquid. Inserting the hand into a bucket of warm water generates a particular sensation of pressure around the wrist (i.e. 'ring') which individuals associate to the perception of liquidity (2). In this case, as cooling cues are not available, individuals rely more on mechanical cues to aid the perception of wetness (3). 
However, in particular situations of local warm-wetness, mechanical cues might be limited. Wearing feminine sanitary products (as well as incontinent products such as diapers) represent one of the real-life situations in which individuals can be exposed to a warm-wet surface and mechanical as well as cooling cues can be limited $(8,9)$. Therefore, in the light of this common real-life situation, the fundamental question we posed would be of practical relevance.

Although the literature on the subjective perception of moisture in clothing is rather extensive within the textile engineering field $(10,11)$, the individual role of thermal and mechanical components in characterising this perception has been rarely investigated $(6,7)$. Thus, there is a need to further the understanding of the psychophysical bases of this complex sensory experience. The aim of this study was to investigate the psychophysical bases of the perception of local skin wetness when the skin of blindfolded individuals was in initial contact with a wet surface with a temperature warmer than the skin. Our expectation is that, if cooling is the main driver for a static wetness perception, when a wet stimulus is applied to the skin with a temperature above the skin temperature, the resulting initial wetness perception will be lower than what we observed in earlier experiments of skin cooling, despite the latter being dry stimuli $(6,7)$.

\section{Material and methods}

\section{Participants}

Seven (five females/two males) healthy university students (age $21 \pm 2$ years) with no history of sensory-related diseases volunteered to participate in this study. All participants gave their informed consent for participation. The study design had been approved by the Loughborough University Ethics Committee and testing procedures were in accordance with the tenets of the Declaration of Helsinki.

\section{Design}

The experimental design was based on the application, in a balanced order, of four different warm stimuli, varying in terms of temperature (i.e. $+4^{\circ} \mathrm{C}$ and $+8^{\circ} \mathrm{C}$ above local skin temperature) and wetness level (i.e. dry or wet). All stimuli were applied on both the bare right upper and lower back of each participant, while participants were resting on a chair in an environmental chamber (set at $22^{\circ} \mathrm{C}$ and $50 \%$ relative humidity). The stimuli were delivered by a thermal probe (Physitemp Instruments Inc., Clifton, NJ, USA) with a contact surface of $25 \mathrm{~cm}^{2}$. The stimulation consisted of a short contact (lasting no longer than $10 \mathrm{~s}$ ) with the probe's surface set at $+4^{\circ} \mathrm{C}$ or $+8^{\circ} \mathrm{C}$ above the individual's local skin temperature [determined using an infrared thermometer (Fluke Corporation, Everett, WA, USA)]. To make the contact with the probe surface dry or wet, test fabrics (100\% cotton) with a surface of $100 \mathrm{~cm}^{2}$ were placed either dry or wet on the probe's surface before the stimulation and fixed by an elastic band. Prior to testing, wet test specimens were soaked for few seconds in $22^{\circ} \mathrm{C}$ water to ensure full saturation and then stored in sealed containers to avoid evaporation. Dry and soaked wet test specimens weighed $1 \mathrm{~g}$ and $3 \mathrm{~g}$ respectively. Wet test specimens' water content was of $0.02 \mathrm{~g} / \mathrm{cm}^{2}$, which was considered acceptable for the purposes of this study as individuals have been previously shown to perceive wetness when in contact with wet surfaces containing an amount of water as little as of $0.0008 \mathrm{~g} / \mathrm{cm}^{2}(1)$.

To ensure that local skin hydration levels would not change significantly during testing procedures (i.e. participants were not sweating due to stress or environmental conditions), the sympathetic skin response was monitored from the beginning and throughout the whole test via galvanic skin conductance (Biopac Systems Inc., Goleta, CA, USA).

\section{Experimental Protocol}

Participants arrived to the laboratory $30 \mathrm{~min}$ before the time scheduled for the test to allow preparation procedures. Male participants wore shorts, socks and trainers whereas female participants wore sport bra, shorts, socks and trainers. Participants were informed only about the body region objected to the stimulation. No information was provided on the type and magnitude of the stimulation to limit any expectation effects. The exact anatomical locations of the areas targeted for stimulation were: $5 \mathrm{~cm}$ upwards the inferior angle of the right scapula (upper back skin site); $5 \mathrm{~cm}$ upwards the right posterior superior iliac spine (lower back skin site). The back was chosen as targeted area for 
stimulation as it has been previously shown to be significantly sensitive to wetness perception (12).

After preparation, participants entered the environmental chamber and $10 \mathrm{~min}$ were allowed for acclimation. During this period, participants were familiarised with the rating scales used to record thermal sensations and wetness perceptions: a modified 11 point thermal sensation scale ( -6 very cold; -4 cold; -2 slightly cool; 0 neutral; +2 slightly warm; +4 warm) and a modified 11 point wetness perception scale $(-6$ dripping wet; -4 wet; -2 slightly wet; 0 neutral; +2 slightly dry; +4 dry) (13). No descriptors were applied to intermediate scores $(-5 ;-3 ;-1 ;+1$; +3 ). We defined the value ' -2 ' (Slightly wet) of the wetness scale as our set threshold to identify a clearly perceived local wetness.

During the test, participants were first asked to rate their thermal sensation and wetness perception before stimulation (i.e. baseline sensation). Then, the required fabric was applied on the thermal probe, which was set to the required relative temperature and then applied (and not moved) to the relevant skin site. As soon as the probe was applied, participants were instructed to report their local and very first sensation and perception, using whatever number in the scales seemed appropriate. The probe was then removed, the skin was gently wiped and its temperature was immediately recorded. This sequence was repeated for each stimulus allowing at least $1 \mathrm{~min}$ in between. Each participant had only one presentation of each stimulus for each body region.

\section{Statistical analysis}

Data were tested for normality of distribution using Shapiro-Wilk test. Skin temperature data were analysed by a 3-way repeated measures analysis of variance (ANOVA), with temperature of the stimulus $\left(+4^{\circ} \mathrm{C}\right.$ vs. $\left.+8^{\circ} \mathrm{C}\right)$, type of stimulus (dry vs. wet), and body region (upper vs. lower back), as within subjects factors. Tukey's post hoc analyses were performed accounting for multiple comparisons and sample size effect. Huynh-Feldt, Geisser-Greenhouse and lowerbound corrections were undertaken to adjust the degrees of freedom for the averaged tests of significance. Thermal and wetness ratings were analysed using a Friedman ANOVA test and post hoc analyses were performed using a Wilcoxon signed-rank tests. All data were analysed using SPSS (IBM, Armonk, NY, USA) and reported as means \pm standard deviation. In all analyses, $P<0.05$ was used to establish significant differences.

\section{Results}

\section{Skin temperature}

Pre-stimulation skin temperature was found to be on average $32.1 \pm 1^{\circ} \mathrm{C}$ for the upper back, and $30.7 \pm 1{ }^{\circ} \mathrm{C}$ for the lower back. No effect of body region was observed on local skin temperature as a result of the stimulation $(P=0.5)$. The $+8^{\circ} \mathrm{C}$ stimuli resulted in a greater increase in local skin temperature $\left(+1.4 \pm 0.8^{\circ} \mathrm{C}\right)$ than the $+4^{\circ} \mathrm{C}$ ones $\left(+0.5 \pm 0.4^{\circ} \mathrm{C}\right)\left(F=16.5_{(1,6)}, P<\right.$ 0.01 ). Dry and wet stimuli resulted in similar relative increases in local skin temperature $(P=0.83)$. Overall, skin temperature always increased on application of the stimuli.

\section{Thermal sensation}

Pre-stimulation thermal sensations ranged from neutral to slightly warm and were found to be not statistically different $(P=0.8)$ between conditions. No effect of body region was found on the thermal sensations recorded during the stimulation $(P=0.9)$. A significant effect of temperature was found, with warmer stimuli resulting in significantly warmer thermal sensations $(Z=-2.04, P<0.05, r=-0.38)$. These varied in a range from $+2 \pm 1\left(+4^{\circ} \mathrm{C}\right.$ stimuli) to $+2.4 \pm 1.5$ ( $+8^{\circ} \mathrm{C}$ stimuli), which corresponded to thermal sensations between slightly warm and warm. A significant effect of type of stimulus (dry vs. wet) was found, with wet stimuli resulting in significantly warmer thermal sensations $(Z=-3.4, P<0.01, r=-0.64)$. These varied in a range from $+1.7 \pm 1$ (dry stimuli) to $+2.7 \pm 1.3$ (wet stimuli), which corresponded to thermal sensations between neutral and warm. A significant interaction between temperature and type of the stimuli was found $\left(\chi^{2}=19.64_{(3,14)}\right.$, $P<0.01)$.

\section{Wetness perception}

Pre-stimulation wetness perceptions ranged from neutral to slightly dry and were found to be not statistically different $(P=0.2)$ (Fig. 1). No effect of body region $(P=0.9)$, nor temperature 
( $P=0.8)$ and type of the stimulus $(P=0.1)$ were found on the wetness perceptions recorded during the stimulation. These ranged from neutral to slightly dry. The threshold we set (point '-2 slightly wet' of the wetness perception scale) to identify a clearly perceived wetness was never reached during any of the four stimulations (Fig. 1). To further elucidate the way warm-dry and warm-wet stimuli were perceived by the participants, with regards to their baseline wetness perception, the average change in the score from pre- to post-stimulation was calculated for each stimulus and then analysed. No effect of body region $(P=0.8)$, nor temperature $(P=1)$ was found on the average change in vote from pre- to post-stimulation, although type of the stimulus showed a trend of a bigger change in the wet stimulus $(P=0.08)$. Changes in vote varied in a range from $-0.6 \pm 2.4$ to $+1 \pm 1.2$ votes (Fig. 1).
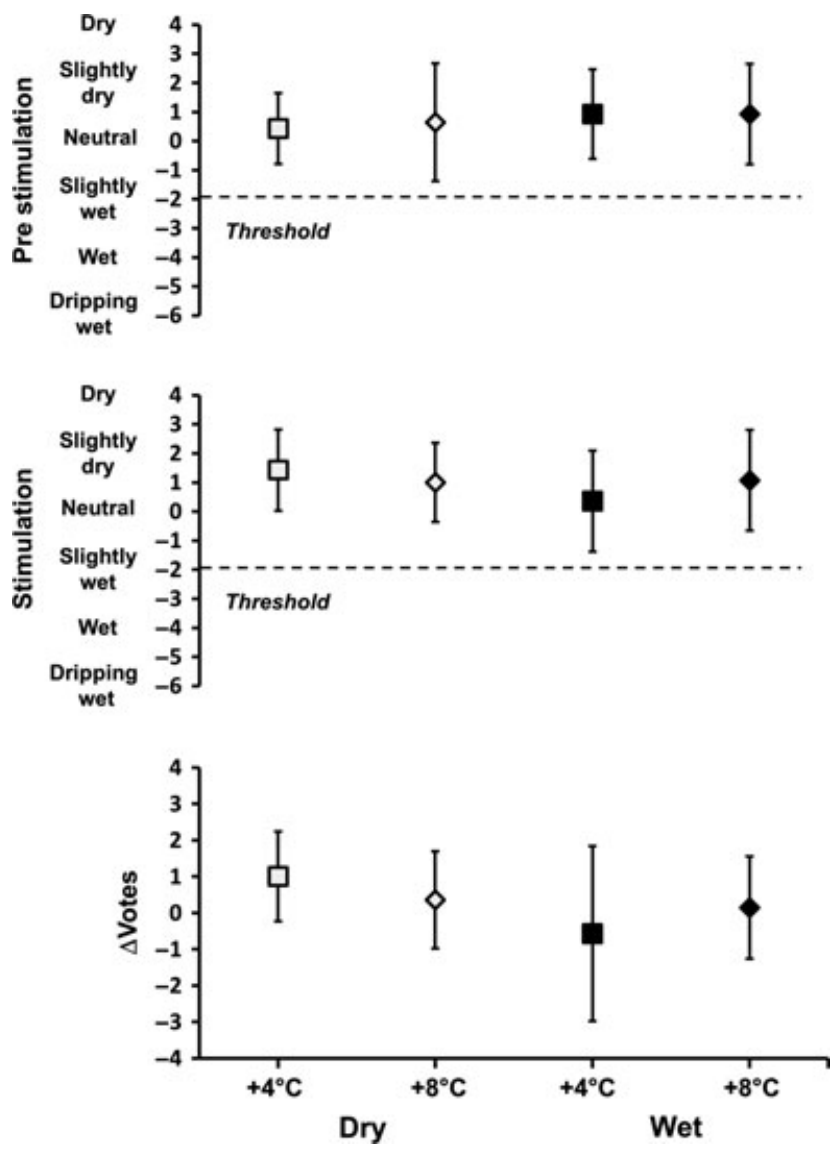

Fig. 1. Wetness perception scores recorded before (pre-stimulation) and during (stimulation) the application of the warm-dry and warmwet stimuli. Average changes in vote (Dvotes) from pre- to post-stimulation are also reported. Data were collapsed over the skin site where the stimulus was applied as no effect of body region (upper vs. lower back) was observed $(P>0.05)$.

\section{Skin conductance}

Average skin conductance values did not significantly change during testing procedures and were observed to remain constantly at a level below $0.5 \mu \mathrm{S}$. These results confirm that no significant variations in the sudomotor activity occurred during the experiment.

\section{Discussion}

The aim of this study was to investigate the psychophysical bases of the perception of local skin wetness. Specifically, it was verified whether individuals would perceive local wet stimuli as wet when these have a temperature warmer than the skin. The outcomes of this study indicated that participants did not perceive that some of the stimuli were wet and did not discriminate between warm-dry and warm-wet stimuli. This represents a novel and interesting finding, as to our knowledge no experimental data are currently available on the subjective thermal and wetness perceptions experienced during the initial contact of the skin with a warm-wet surface.

The possibility that warm sensations might suppress the perception of local wetness seems in line with the findings of our previous study, in which we have demonstrated the importance of experiencing coldness in order to perceive local skin wetness $(6,7)$. We have recently shown that an illusion of local skin wetness can be evoked during the contact with a cold-dry surface inducing a skin cooling rate in a range of $0.14-0.41^{\circ} \mathrm{C} / \mathrm{s}(6,7)$. This observation indicated that it is not the contact of the skin with moisture per se, but rather the integration of specific sensory inputs which seems driving the perception of wetness during the contact with a wet surface (2). Amongst these sensory inputs, experiencing coldness seemed dominant in evoking the perception of local wetness. Although in this study, participants' skin came in contact with a quantity of moisture (i.e. $0.02 \mathrm{~g} / \mathrm{cm}^{2}$ ) far greater than the threshold previously proposed for this perception (i.e. $0.0008 \mathrm{~g} / \mathrm{cm}^{2}$ ) (1), as no skin cooling and thus cold sensations occurred, no perception of local wetness was reported at any time, and warm-wet stimuli were perceived as dry as warm-dry ones. The contact with a moist fabric has been suggested to be perceived as wet, as the presence of moisture leads to higher heat losses from the skin (and thus colder sensations), 
due to the higher thermal conductivity of the wet fabric (14). This phenomenon did not occur in this study as the wet fabric was purposely in contact with a surface warmer than the skin, so that a stronger heat gain, rather than a stronger heat loss, would occur. This design resulted in our participants being unable to clearly perceive local wetness during the initial contact with a warm-wet surface. From a fundamental point of view, this furthers our understanding of the complex sensory integration underpinning the perception of skin wetness. The sensory integration of specific cooling cues seems to critically determine the ability to perceive local skin wetness $(1,6,7)$. This appears to be particularly true when intra- and inter-sensory interactions with other sensory modalities (e.g. mechanical sense and vision) are limited.

However, one should note that the conclusions we propose cannot be generalised to any type of perception of wetness, and should be only limited to the ones resulting from the initial contact with a surface/object. Mechanical inputs could have a role as critical as thermal inputs in characterising this perception, particularly when cooling cues are not available (3). If thermal cues are limited, individuals seem to rely more on mechanical sensations, such as 'stickiness', to characterise their perception of wetness when e.g. wearing wet clothes (15) or manipulating wet surfaces (16).
The findings of this study have an applied significance, as they could contribute to the design and optimisation of sanitary products (e.g. diapers) for personal and patients care. As the occurrence of wetness is a common event when wearing these products, the fact that warm-wetness might be sometimes difficult to perceive highlights the need to develop systems for alerting of the occurrence of wetness (9). This could increase the awareness of local skin wetness, thus improving personal care (17), particularly within clinical contexts.

\section{Conclusion}

Warm temperature stimuli have been shown to suppress the perception of skin wetness during initial contact with a wet surface. Hence, we conclude that it is not the contact of the skin with moisture per se, but rather the integration of particular sensory inputs which drives the perception of skin wetness during the initial contact with a wet surface. When the contribution of other sensory inputs (i.e. dynamic pressure and vision) is limited, experiencing coldness could be the primary driver of the perception of wetness.

\section{Acknowledgements}

The present research was done in the context of an industry co-funded PhD.

\section{References}

1. Ackerley R, Olausson H, Wessberg J, McGlone F. Wetness perception across body sites. Neurosci Lett 2012; 522: 73-77.

2. Bentley I. The synthetic experiment. Am J Psychol 1900; 11: 405425.

3. Bergmann Tiest WM, Kosters ND, Kappers AML, Daanen HAM. Haptic perception of wetness. Acta Psychol 2012; 141: 159-163.

4. Clark R, Edholm O. Man and his thermal environment. London: E. Arnold; 1985: 253.

5. Cappe C, Rouiller EM, Barone P. Multisensory anatomical pathways. Hear Res 2009; 258: 28-36.

6. Filingeri D, Redortier B, Hodder S, Havenith $G$. The role of decreasing contact temperatures and skin cooling in the perception of skin wetness. Neurosci Lett 2013; 551: 65-69.
7. Filingeri D, Redortier B, Hodder S, Havenith G. Thermal and tactile interactions in the perception of local skin wetness at rest and during exercise in thermo-neutral and warm environments. Neuroscience 2014; 258: 121-130.

8. Farage M, Meyer S, Walter D. Evaluation of modifications of the traditional patch test in assessing the chemical irritation potential of feminine hygiene products. Skin Res Technol 2004; 10: 73-84.

9. Daanen HAM. Method and system for alerting the occurrence of wetness. EP Patent 2009; 2,110,108. Available at: http://www.freepatentsonline.com/EP2110108.html

10. Sweeney MM, Branson DH. Sensorial comfort: part I: a psychophysical method for assessing moisture sensation in clothing. Text Res J 1990; 60: 371-377.

11. Sweeney MM, Branson DH. Sensorial comfort: part II: a magnitude estimation approach for assessing moisture sensation 1. Text Res J 1990; 60: 447-452.

12. Fukazawa T, Havenith G. Differences in comfort perception in relation to local and whole body skin wettedness. Eur J Appl Physiol 2009; 106: 15-24.

13. Olesen B, Brager G. A better way to predict comfort: the new ASHRAE standard 55-2004. ASHRAE Journal 2004; 8: 20-26.

14. Niedermann R, Rossi R. Objective and subjective evaluation of the human thermal sensation of wet fabrics. Text Res J 2012; 82: 374-384.

15. Sukigara S, Niwa M. Analysis of "wet" sensation for lingerie fabrics. Int J Clothing Sci Technol 1997; 9: 214-219.

16. Essick GK, McGlone F, Dancer C, Fabricant D, Ragin Y, Phillips N, Jones T, Guest S. Quantitative assessment of pleasant touch. Neurosci Biobehav Rev 2010; 34: 192-203. 
Filingeri et al.

17. Akin F, Lemmen J. A refined method to evaluate diapers for effectiveness in reducing skin hydration using the adult forearm. Skin Res Technol 1997; 3: 173-176.
Address:

D. Filingeri

Environmental Ergonomics Research Centre

Loughborough Design School
Loughborough University Loughborough, LE11 3TU UK

Tel: +44 (0)1509 223022

e-mail: D.Filingeri@lboro.ac.uk 